\title{
Morpholino-mediated exon inclusion for SMA
}

Haiyan Zhou ${ }^{1}$ and Francesco Muntoni ${ }^{1 *}$

${ }^{1}$ The Dubowitz Neuromuscular Centre, Molecular Neurosciences Session, Developmental

Neurosciences Programme, Great Ormond Street Institute of Child Health, University College

London. 30 Guilford Street, London WC1N 1EH. United Kingdom.

E-mail: f.muntoni@ucl.ac.uk 


\section{Abstract}

The application of antisense oligonucleotides (AONs) to modify pre-messenger RNA splicing has great potential for treating genetic diseases. The strategies used to redirect splicing for therapeutic purpose involve the use of AONs complementary to splice motifs, enhancer or silencer sequences. AONs to block intronic splicing silencer motifs can efficiently augment exon 7 inclusion in survival motor neuron 2 (SMN2) gene and have demonstrated robust therapeutic effects in both pre-clinical studies and clinical trials in spinal muscular atrophy (SMA), which lead to a recently approved drug. AONs with phosphoroamidate morpholino (PMO) backbone have shown target engagement with restoration of the defective protein in Duchenne muscular dystrophy (DMD) and their safety profile lead to a recent conditional approval for one DMD PMO drug. PMO AONs are also effective in correcting SMN2 exon 7 splicing and rescuing SMA transgenic mice. Here we provide the details of methods that our lab has used to evaluate PMO-mediated SMN2 exon 7 inclusion in the in vivo studies conducted in SMA transgenic mice. The methods comprise mouse experiment procedures, assessment of PMOs on exon 7 inclusion at RNA levels by reverse transcription (RT-) PCR and quantitative real-time PCR. In addition, we present methodology for protein quantification using western blot in mouse tissues, on neuropathology assessment of skeletal muscle (muscle pathology and neuromuscular junction staining) as well as behaviour test in the SMA mice (righting reflex).

Key words: Antisense oligonucleotide, PMO, alternative splicing, exon inclusion, SMN2, SMA, transgenic mouse model, skeletal muscle pathology, neuromuscular junction, motor neurons. 


\section{Introduction}

Pre-mRNA splicing is the editing procedure to remove introns from the nascent precursor messenger RNA (pre-mRNA) transcript in order to form a mature messenger RNA (mRNA) where only the coding sequences, exons, remain to be translated into protein. Alternative splicing is a regulated process that leads to a single gene coding for multiple proteins. It is a major contribution to the regulation of gene expression based on the fact that almost all, $94 \%$ of protein coding genes, contain introns and of these $90 \%$ show significant levels of alternative splicing. Using RNA-based technology such as antisense oligonucleotide (AON) to modulate gene expression by regulating pre-mRNA splicing has become a promising therapeutic approach where alternative splicing is the target.

The strategy used to redirect splicing for therapeutic purpose involves the use of AONs complementary to splice motifs, enhancer or silencer sequences, including exonic and intronic splicing enhancers (ESEs/ISEs) or silencers (ESSs/ISSs). While AONs to target ESEs are effective in promoting exon-skipping, such as in Duchenne muscular dystrophy [1], AONs complementary to an ISS have shown significant effect on augmenting exon-inclusion in another neuromuscular condition, spinal muscular atrophy (SMA).

The genetic defect in patients with SMA is the loss of survival of motor neuron (SMN) protein, caused in $95 \%$ of cases by homozygous deletions of the survival of motor neuron 1 (SMN1) gene [2]. $S M N 2$ is the paralogue gene to $S M N 1$ and is intact in the general population. However, due 
to the alternative splicing of exon 7 in $S M N 2$, the $S M N 2$ gene cannot fully compensate for the loss of the SMN1 gene, and only $10 \%$ of its transcripts can correctly splice exon 7 [3].

The alternative splicing of the $S M N 2$ gene transcript is due to a $C>T$ single-nucleotide substitution in exon 7 that, without altering the amino acid encoded, disrupts an ESE in that exon and causes it to be excluded from the mature $S M N 2$ transcript during splicing [4]. In the SMN2 gene, a few regulatory elements have been shown to modulate the alternative splicing of exon 7. These include element 1 in intron 6, an SF2/ASF (alternative splicing factor/splicing factor-2) or hnRNP (heterogeneous nuclear ribonucleoprotein) A1-binding site in exon 7 (the site containing the $C>$ T substitution), the Tra2- $\beta 1$-binding site in exon 7, the $3^{\prime}$ cluster at the $3^{\prime}$ end of exon7, the intronic splicing silencer N1 (ISS-N1) and ISS-N2 in intron 7, and hnRNP A1binding sites in intron 7. Among these regulatory sites, AONs targeting ISS-N1 showed the most significant effect on augmenting exon 7 inclusion in SMN2 gene and restoring SMN protein in vitro in cellular models, and in vivo in different SMA mouse models [5-8]. So far there are four different chemical modifications in the ISS-N1-targeting AONs have been tested in preclinical studies in SMA transgenic mice, including 2'-O-Methyl, 2'-0-2-methoxyethyl phosphorothioate (MOE), phosphoroamidate morpholino (PMO) and tricyclo-DNA AONs $[5,7,9,10]$. Spinraza (Nusinersen), an AON drug targeting ISS-N1 in MOE backbone, is so far the only drug that has been approved by the US Food and Drug Administration (FDA) and the European Medical Association (EMA) for the treatment of SMA and is now commercially available in several countries for patients with all types of SMA [11]. The PMO backbone has been extensively studied in pre-clinical and clinical studies in another neuromuscular condition, Duchenne muscular dystrophy, and demonstrated excellent safety with no drug-related adverse effect in 
clinical trials [12]. The excellent safety profile of PMOs has also been indicated in preclinical studies in non-human primates and rodents when long-term and high dose of PMOs were delivered $[5,13]$. We and the others have shown the striking and reliable therapeutic effect of PMOs in SMA on correcting SMN2 exon 7 splicing and improving phenotypes and survivals in transgenic mouse models $[5,6,14-17]$. We describe here the methods generally used to test the efficiency of PMOs on exon-inclusion in vivo in SMA mouse models.

\section{Materials}

\subsection{Morpholino antisense oligomers}

1. Morpholinos are synthesized commercially by Gene Tools, LLC (www.genetools.com).

2. $1 \mathrm{mM}$ stock solution is prepared in distilled water.

3. Store Morpholino stock solutions and working solutions at room temperature (see Note 1).

4. Ensure the oligomer is completely dissolved when making stock solution.

5. If some solid remains or Morpholinos are stored cold or frozen, heat the solution for 10 minutes at $65^{\circ} \mathrm{C}$, briefly vortex and cool to room temperature.

\subsection{SMA transgenic mice and procedures in neonatal mice}

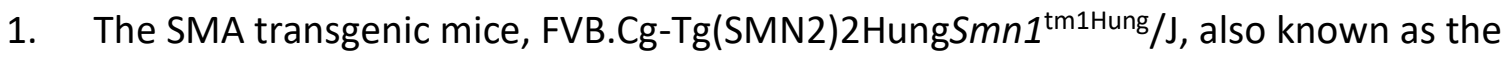
Taiwanese SMA mouse model, is commercially available from the Jackson Laboratory (TJL005058).

2. Litters born from the breeding pairs of mice with genotype $(\mathrm{SMN} 2)_{2}{ }^{+/+} ; \mathrm{smn}^{-/-}$ (mild type III SMA mice) crossed with the genotype $\mathrm{smn}^{+/-}$(heterozygous wild- 
type) consist of $50 \%$ wild-type "heterozygous" progeny with genotype $(\mathrm{SMN} 2)_{2}{ }^{+/}$; $\mathrm{smn}^{+/-}$and 50\% severe type I SMA progeny with genotype (SMN2) $2^{+--} ; \mathrm{smn}^{-/-}($see Note 2).

3. $10 \mu \mathrm{l}$ capillary glass micropipettes (Drummond Scientific Company) with flamepolished end.

4. $\quad 0.9 \%$ saline.

\subsection{Analysing PMO activity on exon 7 inclusion at RNA levels in tissues from SMA mice}

1. Precellys tissue Homogenizer (see Note 3).

2. RNeasy mini kit and QIAshredder columns.

3. SuperScript ${ }^{\mathrm{TM}}$ III First-Strand cDNA Synthesis kit.

4. Human SMN1/2-specific primers for RT-PCR: Forward, 5'-CTC CCA TAT GTC CAG ATT CTC TT-3'; Reverse, 5'-CTA CAA CAC CCT TCT CAC AG-3'. The PCR product is $505 \mathrm{bp}$ for the full-length $S M N 1 / 2$ transcript and $451 \mathrm{bp}$ for the $\triangle 7$ SMN2 transcript.

5. $10 \times$ PCR buffer, $10 \mathrm{mM}$ dNTPs, $50 \mathrm{mM} \mathrm{MgCl}$, Taq DNA polymerase $(5 \mathrm{U} / \mu \mathrm{l}), 10 \mu \mathrm{M}$ forward primer, $10 \mu \mathrm{M}$ reverse primer and nuclease-free water.

6. $\quad 0.2 \mathrm{ml}$ nuclease-free microcentrifuge tubes.

7. $1 \times$ Tris/Borate/EDTA (TBE) buffer, $1.5 \%$ Agarose gel, SYBR safe DNA gel stain, 100bp DNA ladder.

8. StepOne real-time PCR system (Applied Biosystems)

9. 96-well real-time PCR plate and sealing film (for quantitative real-time PCR).

10. Universal SYBR Green MasterMix (with Rox) (2x). 
11. Template cDNA diluted $20 \times$ for $q P C R$ reaction.

12. qPCR primers $10 \mathrm{pmol} / \mu \mathrm{L}$ each.

13. Human-specific full-length SMN2 primers (forward, 5'-ATA CTG GCT ATT ATA TGG GTT TT-3'; reverse, 5'-TCC AGA TCT GTC TGA TCG TTT C-3' [133 bp]) and humanspecific $\triangle 7$ SMN2 primers [forward, 5'-TGG ACC ACC AAT AAT TCC CC-3'; reverse, 5'-ATG CCA GCA TTT CCA TAT AAT AGC C-3' [125 bp]).

\subsection{Measuring human $S M N$ protein by western blotting in tissues from SMA mice}

1. Precellys tissue Homogenizer (see Note 3).

2. Western blot sample buffer: $0.25 \%$ SDS, $75 \mathrm{mM}$ Tris- $\mathrm{HCl}(\mathrm{pH} 6.8)$.

3. Protease inhibitor cocktail.

4. Pierce BCA (Bicinchoninic acid) Protein Assay Kit.

5. $\quad$ PBST (PBS, pH 7.4, 0.1\% Tween 20)

6. Mini gel tank and blot module set

7. NUPAGE $10 \%$ Bis-Tris precast gels, LDS sample buffer $(4 \times)$, SDS running buffer (20x), antioxidant, sample reducing buffer, transfer buffer (20x), methanol.

8. Protein ladder.

9. PVDF membrane.

10. Whatman filter paper.

11. Odyssey blocking buffer for PVDF membrane blocking.

12. Antibodies: mouse anti-SMN monoclonal antibody (BD Transduction Laboratories), mouse anti- $\beta$-tubulin monoclonal antibody (Sigma), IRDye 800CW-conjugated goat anti-mouse secondary antibody (Li-Cor). 
13. Odyssey imaging instrument to quantify western blot signals.

\subsection{Skeletal muscle histopathology in Haematoxylin and Eosin (H\&E) staining}

1. Dissecting tools (scissors and forceps)

2. Gum Tragacanth, Iso-pentane, liquid Nitrogen, corks.

3. Superfrost glass slides and coverslips.

4. Harris Haematoxylin, Eosin yellowish, $100 \%$ ethanol, $70 \%$ ethanol, Xylene, DPX mountant.

5. Light microscope.

\subsection{Neuromuscular junction staining}

1. Dissecting tools (scissors and forceps) and microscope.

2. PBS and Petri dish.

3. $4 \%$ Paraformaldehyde in PBS.

4. Blocking and permeabilizing buffer ( $5 \%$ goat serum and $1 \%$ Triton $\mathrm{X}-100$ in PBS).

5. Antibodies: rabbit polyclonal anti-neurofilament antibody (NF200, Sigma), and rabbit polyclonal anti-synaptophysin antibody (Synaptic Systems), rhodamine- $\alpha-$ bungarotoxin ( $\alpha-B T)$ (Life Technology) and Alexa Fluor 488 goat anti-rabbit IgG (Life Technology).

6. Glass slides and coverslips.

7. Hydromount mounting medium.

8. Fluorescence microscope and confocal microscope.

\section{Methods}

\subsection{Injection of PMOs in neonatal mice}


1. PMOs are injected into newborn mice by subcutaneously (SC) or intracerebroventricular (ICV) injections (see Note 4).

2. ICV injection: the injection sites are approximately $1 \mathrm{~mm}$ lateral from the sagittal suture and $1 \mathrm{~mm}$ anterior to the coronal suture (Fig. 1, see Note 5) (6). Slowly insert the glass capillary through the skin to an approximately $2 \mathrm{~mm}$ depth until the instant resistance from the frontal bone disappears. Alternatively, the needle can be marked with tape at $2 \mathrm{~mm}$ from the tip to ensure the injection is $2 \mathrm{~mm}$ deep. Push in the plunge slowly to complete the injection. The injection volume is no more than $10 \mu \mathrm{l}$ in total into both ventricles.

3. Subcutaneous (SC) injection: Use fingers to lift the loose skin at the back between shoulder blades. Direct needle into the centre of the skin and slowly inject solutions. 30 gauge needle and $1 \mathrm{ml}$ syringe can be used for SC injection in neonatal mice, in addition to capillary glass micropipettes.

4. Sacrifice PMO-treated pups at the age required. Tissues are dissected, snap frozen on dry ice and stored at $-80 \mathrm{C}$ until the next experiment.

\subsection{Splicing assay of PMOs on exon 7 inclusion in tissues from SMA mice}

1. Frozen tissues are placed in RLT lysis buffer in $2 \mathrm{ml}$ tubes containing $2.8 \mathrm{~mm}$ ceramic (zirconium oxide) beads and homogenized using Precellys tissue Homogenizer. (see Note 3)

2. Extract RNA from the tissues using RNeasy Mini Kit according to manufacturer's instruction. 
3. Reverse transcription: the cDNA is synthesized from 500ng RNA using the SuperScript ${ }^{\mathrm{TM}}$ III First-Strand cDNA Synthesis kit according to manufacturer's instruction.

4. Reverse-transcript PCR of human SMN2: Perform PCR according to standard procedures. Use $1 \mu \mathrm{l} \mathrm{cDNA}$ in a $25 \mu \mathrm{l}$ PCR reaction with 500 pmol of each primer (see subheading 2.3.4), $200 \mu \mathrm{M}$ of dNTPs, $1.5 \mathrm{mM} \mathrm{MgCl}_{2}, 2.5$ units of Taq polymerase and 1× PCR buffer. The PCR amplification program is as follows: 1 cycle with 3 minutes at $94^{\circ} \mathrm{C}$ (initial denaturation), 25-30 subsequent cycles of 30 seconds at $94^{\circ} \mathrm{C}$ (denaturation), 30 seconds at $55^{\circ} \mathrm{C}$ (annealing), and 30 seconds at $72^{\circ} \mathrm{C}$ (extension) and a final 10 minutes extension at $72^{\circ} \mathrm{C}$. Check an aliquot of the PCR product (5-10 $\mu$ l) by $1.5 \%$ agarose gel electrophoresis and SYBR safe DNA stain using an UV transilluminator. The top band of $505 \mathrm{bp}$ is the full-length SMN2 product. The lower $451 \mathrm{bp}$ band is the product with exon 7 skipping ( $\triangle 7 S M N 2$ ) (Fig. 2a).

5. Quantitative real-time PCR of human-specific full-length and $\triangle 7 S M N 2$ transcripts: product specific primers (see subheading 2.3.13), cDNA and $1 \times$ PCR Mastermix are mixed in a $20 \mu \mathrm{PCR}$ reaction. The program includes activation at $95^{\circ} \mathrm{C}$ for 5 minutes, 40 cycles of $95^{\circ} \mathrm{C}$ for 3 seconds, and $60^{\circ} \mathrm{C}$ for 1 minute. Quantification is based on concurrent standard curves produced from serial dilutions of cDNA from saline treated control SMA mouse. The cycle at which the amount of fluorescence is above the threshold $(\mathrm{Ct})$ is detected. The ratios of full-length SMN2 to $\triangle 7 \mathrm{SMN} 2$ 
(FL/ $\Delta 7$ ) from PMO-treated mice samples are normalized, taking the ratio of saline control of mouse tissues as 1.0.

6. Western blot: frozen tissues are placed in protein sampling buffer and homogenized using Precellys tissue Homogenizer kit (see Note 3). Centrifuge at $12,000 \times g$ and $4^{\circ} \mathrm{C}$ for 10 minutes. Transfer the supernatant to a fresh tube. The protein concentration is measured by a NanoDrop spectrophotometer using the Pierce BCA Protein Assay Kit according to the manufacturer's instructions. Fifty micrograms of total protein extracted from mouse tissues are separated by electrophoresis on a $10 \%$ NuPAGE Bis-Tris precast gels in running buffer, and electro-transferred to a PVDF membrane in transfer buffer. Block the PVDF membrane for 1 hour in blocking buffer and incubate with the primary antibodies on a shaker at $4^{\circ} \mathrm{C}$ overnight. Wash the PVDF membrane $3 \times 10$ minutes in PBST. Incubate the PVDF membrane with fluorescence second antibody for 1 hour at room temperature. Wash $3 \times 10$ minutes in PBST and detect bands using Odyssey Imaging system. (Fig. 2b)

\subsection{H\&E staining in skeletal muscle biopsies}

1. Fresh muscles dissected from mice are bisected transversely and mounted in tragacanth gum on cork squares.

2. The muscles are frozen in liquid nitrogen-cooled iso-pentane and stored at $-80^{\circ} \mathrm{C}$ freezer. 
3. Cut cryosections at $8 \mu \mathrm{m}$ directly on uncoated glass slide and dry for 10 minutes at room temperature before staining. Or the slides can be stored at $-80^{\circ} \mathrm{C}$ until the staining.

4. H\&E staining: stain sections with Haematoxylinn for 30 seconds. Rinse excess stain in tap water until nuclei turn blue. Soak slides in $70 \%$ ethanol for 3 minutes then Eosin for 15 seconds. Dehydrate slides in $100 \%$ ethanol for 3 minutes (repeat $\times 3$ ). Clear slides with Xylene wash for 3 minutes (repeat $\times 3$ ). Mount slides with DPX mountant and coverslips.

5. Images are acquired using light microscope, digital camera and image capture software. Digital slide scanner is optimal if possible.

6. The histopathological features of muscle biopsies are identified using image analysis software (e.g. Image J).

\subsection{Neuromuscular junction staining}

1. Skeletal muscles are dissected and fixed in $4 \%$ paraformaldehyde on ice for 4 hours.

2. The fixed muscle tissues are washed thoroughly in PBS.

3. Block muscle fibres in $5 \%$ goat serum and $1 \%$ Triton $\mathrm{X}-100$ in PBS for 1 hour at room temperature.

4. Incubate muscle fibres in primary antibodies (anti-Neurofilament and antisynaptophysin) diluted in blocking buffer at $4^{\circ} \mathrm{C}$ overnight (see Note 6). 
5. Quickly wash in PBS for 3 times and then incubate in PBS buffer with Alexa Fluor 488 goat anti-rabbit IgG and rhodamine- $\alpha$-bungarotoxin for 4 hours at room temperature.

6. Muscles are vigorously washed in PBS before being whole-mounted in hydromount mounting medium.

7. Large muscles (e.g. tibialis anterior and gastrocnemius) need further dissection to a smaller size suitable for mounting.

8. Visualize slides on fluorescence microscope (Fig. 3).

9. To detect and quantify NMJ pathology, confocal microscope is required to get highresolution z-stack project images.

10. A minimum of $30 \mathrm{NMJs}$, ideal to $100-200 \mathrm{NMJs}$, are quantified per muscle on size and maturation status of the motor endplates and morphology of denervation/innervation.

\subsection{SMA mouse behaviour test- righting reflex}

1. Place mouse on its back on a bench top with all four paws up in the air.

2. Release fingers and start time counting with a timer.

3. Count the time when the mouse has flipped over on its stomach with all four paws touching the ground (see Note 7 and 8).

4. Repeat procedure three times for each mouse, with at least 5 min recovery period between tests.

5. The maximum recording time is 30 seconds (see Note 9 ).

\section{Notes}


1. Morpholino should be stored at room temperature. Storage at $4^{\circ} \mathrm{C}$ or freezer will cause precipitation and decrease in activity. Long-term storage at room temperature may also cause aggregation over time. This can be resolved by heating $5 \mathrm{~min}$ at $65^{\circ} \mathrm{C}$ or by autoclaving. PMO is very heat stable.

2. The Taiwanese male mice have impaired reproductive organ development and reduced fertility [18]. They usually take longer pairing time than the wild type mice. It is therefore recommended to pair the female type III SMA mice $\left((S M N 2) 2_{2}^{+/+} ; s m n^{-/-}\right)$with the male heterozygous wild-type $\left(\left(\mathrm{SMN}_{2}\right)_{2} 2^{-/-} ; s \mathrm{mn}^{-/-}\right)$to produce the severe type I SMA mice $\left(\left(\mathrm{SMN}_{2}\right)_{2}{ }^{+/-} ; \mathrm{smn}^{-/-}\right)$for PMO evaluation. It is recommended that the breeders need to be refreshed every 6 months, especially for the type III SMA breeders. The first breeding in female SMA mice should take place no later than 12 weeks old.

3. If the Precellys tissue Homogenizer is not available, frozen tissue can be pulverized in liquid nitrogen with mortar and pestle, before adding the RLT Iysis buffer or protein sampling buffer and proceeding with RNA/protein isolation.

4. A very narrow therapeutic window has been previously described in the severe SMA mice $[19,20]$. It is therefore recommended that PMO treatment is delivered during the pre-symptomatic stage in order to achieve a maximal therapeutic benefit. In our experience, the best efficacy is gained when PMO injection is administered at 1-day or 2-days old.

5. When practice ICV injection for the first time, a dye (i.e Fast Green FCF or trypan blue) can be mixed with solution to indicate if the solution is correctly delivered into lateral ventricles. The injection sites of ICV in neonatal mice are not consistent in different 
publications. We have compared a number of the reported injection sites and the one we report here gives the highest successful rate without any adverse effect such as cerebral haemorrhage.

6. It is important to use antibodies to stain both neurofilament and synaptophysin in the staining of NMJ in order to get the detail of nerve ends in endplates in NMJ.

7. In righting reflex test, the test is not over if the mouse has one paw underneath its body. Count the time when all four paws touch the ground.

8. In behaviour test there could be high variation between litters. To reduce it and keep experiments consistent, it is recommended to keep the litter size between 5-10 pups per litter. Litter under 5 pups should not be included for phenotyping as they usually receive more maternal care and may present milder phenotype. Litter over 10 should be reduced to the size of 10 to avoid competition for feeding and care.

9. Some protocols may set the time limit at 60 seconds. In our experience in the Taiwanese SMA mouse models, mice failed in righting themselves in 30 seconds were usually at severe condition and they could hardly right later. We therefore set 30 seconds as the limit.

\section{Acknowledgement}

This work was supported by University College London, UK Medical Research Council (MRC) and SMA-Europe. 


\section{Reference List}

1. Dunckley MG, Manoharan M, Villiet P, Eperon IC, Dickson G (1998) Modification of splicing in the dystrophin gene in cultured Mdx muscle cells by antisense oligoribonucleotides. Hum Mol Genet 7: 1083-1090

2. Lefebvre S, Burglen L, Reboullet S, Clermont O, Burlet P, Viollet L et al (1995) Identification and characterization of a spinal muscular atrophy-determining gene. Cell 80: 155-165

3. Lorson CL, Hahnen E, Androphy EJ, Wirth B (1999) A single nucleotide in the SMN gene regulates splicing and is responsible for spinal muscular atrophy. Proc Natl Acad Sci U S A 96: 6307-6311

4. Cartegni L, Krainer AR (2002) Disruption of an SF2/ASF-dependent exonic splicing enhancer in SMN2 causes spinal muscular atrophy in the absence of SMN1. Nat Genet 30: 377-384

5. Porensky PN, Mitrpant C, McGovern VL, Bevan AK, Foust KD, Kaspar BK et al (2012) A single administration of morpholino antisense oligomer rescues spinal muscular atrophy in mouse. Hum Mol Genet 21: 1625-1638

6. Zhou H, Janghra N, Mitrpant C, Dickinson R, Anthony K, Price L et al (2013) A Novel Morpholino Oligomer Targeting ISS-N1 Improves Rescue of Severe SMA Transgenic Mice. Hum Gene Ther 24: $331-342$

7. Hua Y, Sahashi K, Hung G, Rigo F, Passini MA, Bennett CF et al (2010) Antisense correction of SMN2 splicing in the CNS rescues necrosis in a type III SMA mouse model. Genes Dev 24: 16341644 
8. Hua Y, Sahashi K, Rigo F, Hung G, Horev G, Bennett CF et al (2011) Peripheral SMN restoration is essential for long-term rescue of a severe spinal muscular atrophy mouse model. Nature 478: $123-126$

9. Williams JH, Schray RC, Patterson CA, Ayitey SO, Tallent MK, Lutz GJ (2009) Oligonucleotidemediated survival of motor neuron protein expression in CNS improves phenotype in a mouse model of spinal muscular atrophy. J Neurosci 29: 7633-7638

10. Robin V, Griffith G, Carter JL, Leumann CJ, Garcia L, Goyenvalle A (2017) Efficient SMN Rescue following Subcutaneous Tricyclo-DNA Antisense Oligonucleotide Treatment. Mol Ther Nucleic Acids 7: 81-89

11. Finkel RS, Mercuri E, Darras BT, Connolly AM, Kuntz NL, Kirschner J et al (2017) Nusinersen versus Sham Control in Infantile-Onset Spinal Muscular Atrophy. N Engl J Med 377: 1723-1732

12. Cirak S, Arechavala-Gomeza V, Guglieri M, Feng L, Torelli S, Anthony K et al (2011) Exon skipping and dystrophin restoration in patients with Duchenne muscular dystrophy after systemic phosphorodiamidate morpholino oligomer treatment: an open-label, phase 2 , dose-escalation study. Lancet 378: 595-605

13. Sazani P, Ness KP, Weller DL, Poage DW, Palyada K, Shrewsbury SB (2011) Repeat-dose toxicology evaluation in cynomolgus monkeys of AVI-4658, a phosphorodiamidate morpholino oligomer (PMO) drug for the treatment of duchenne muscular dystrophy. Int J Toxicol 30: 313321 
14. Mitrpant C, Porensky P, Zhou H, Price L, Muntoni F, Fletcher S et al (2013) Improved antisense oligonucleotide design to suppress aberrant SMN2 gene transcript processing: towards a treatment for spinal muscular atrophy. PLoS One 8: e62114

15. Nizzardo M, Simone C, Salani S, Ruepp MD, Rizzo F, Ruggieri M et al (2014) Effect of combined systemic and local morpholino treatment on the spinal muscular atrophy Delta7 mouse model phenotype. Clin Ther 36: 340-356

16. Hammond SM, Hazell G, Shabanpoor F, Saleh AF, Bowerman M, Sleigh JN et al (2016) Systemic peptide-mediated oligonucleotide therapy improves long-term survival in spinal muscular atrophy. Proc Natl Acad Sci U S A 113: 10962-10967

17. Osman EY, Miller MR, Robbins KL, Lombardi AM, Atkinson AK, Brehm AJ et al (2014) Morpholino antisense oligonucleotides targeting intronic repressor Element1 improve phenotype in SMA mouse models. Hum Mol Genet 23: 4832-4845

18. Ottesen EW, Howell MD, Singh NN, Seo J, Whitley EM, Singh RN (2016) Severe impairment of male reproductive organ development in a low SMN expressing mouse model of spinal muscular atrophy. Sci Rep 6: 20193

19. Robbins KL, Glascock JJ, Osman EY, Miller MR, Lorson CL (2014) Defining the therapeutic window in a severe animal model of spinal muscular atrophy. Hum Mol Genet 23: 4559-4568

20. Zhou H, Meng J, Marrosu E, Janghra N, Morgan J, Muntoni F (2015) Repeated low doses of morpholino antisense oligomer: an intermediate mouse model of spinal muscular atrophy to explore the window of therapeutic response. Hum Mol Genet 24: 6265-6277 
Figures and legends

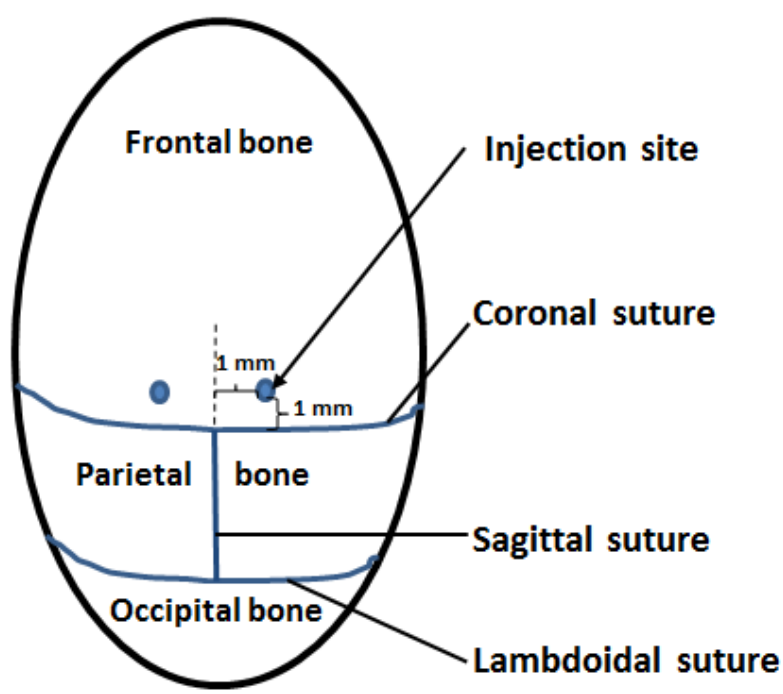

Fig. 1 Schematic illustration of mouse skull sutures and bones. The ICV injection site is $1 \mathrm{~mm}$ from the sagittal suture and $1 \mathrm{~mm}$ anterior to the coronal suture.
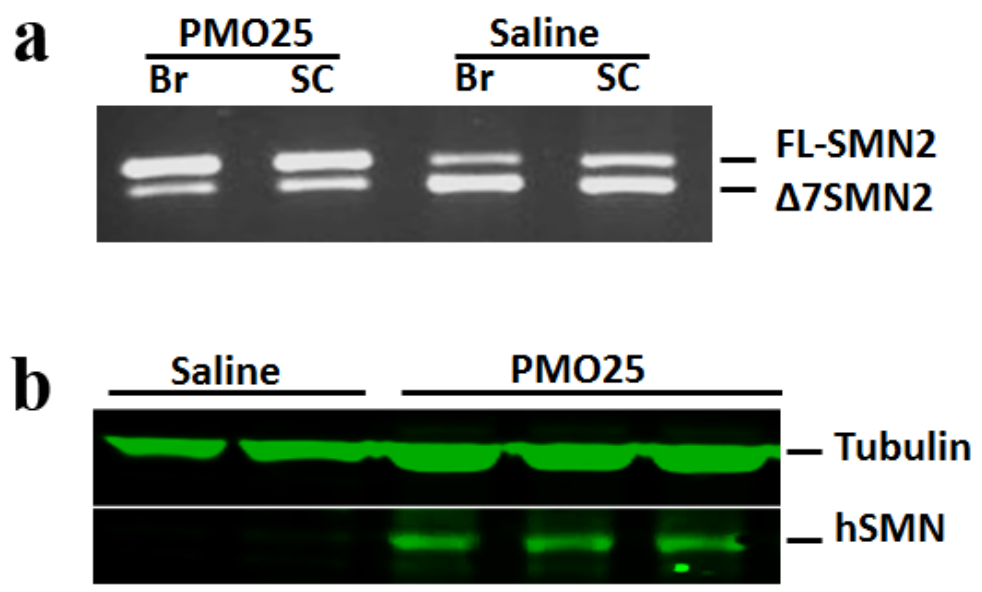

Fig. 2 PMO activity on exon 7 inclusion in tissues from SMA mice. Severe SMA mice were received a single subcutaneous injection of PMO25, a 25-mer PMO targeting ISS-N1, at the dose of $40 \mu \mathrm{g} / \mathrm{g}$ on postnatal day 0. SMA mice received same volume of saline were used as control. 
Brain and spinal cord tissues were collected 7 days after the injection. a. Representative picture of reverse-transcription PCR shows the expression of the full-length SMN2 and $\triangle 7$ SMN2 transcripts in brain and spinal cords after the treatment. b. Representative picture of western blotting shows the restoration of human SMN protein in spinal cord tissues of PMO25-treated SMA mice (protein samples from 3 PMO25-treated SMA mice) compared to saline-treated SMA mice (protein samples from 2 saline-treated SMA mice).
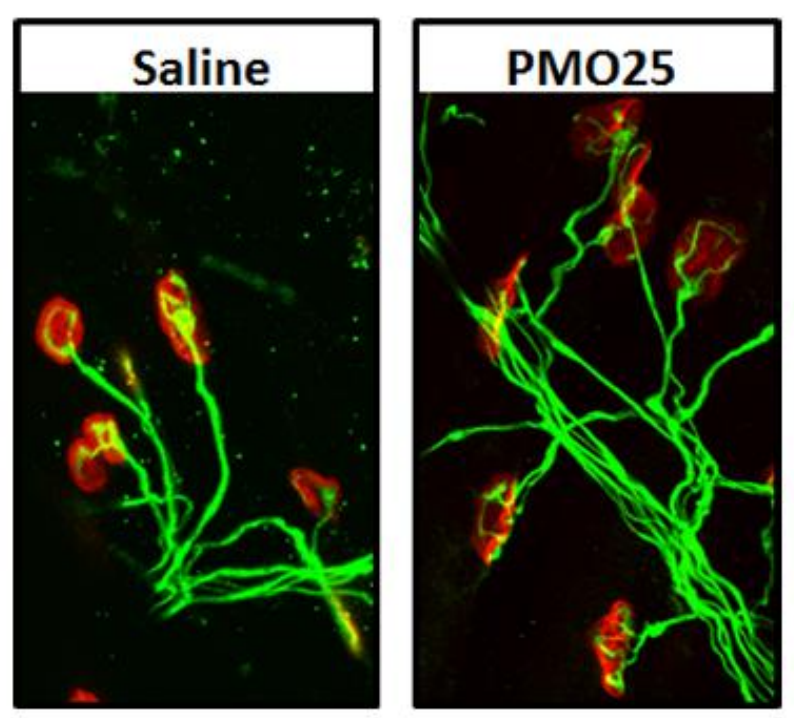

Fig. 3 Representative pictures of NMJs staining in the tibialis anterior muscles collected from 10 days old SMA mice. The SMA mice received a single dose of $40 \mu \mathrm{g} / \mathrm{g}$ PMO25 at PNDO by subcutaneous injection. Saline treated mice were used as controls. The endplates were stained with rhodamine- $\alpha$-bungarotoxin (in red) and nerve fibres were stained with antibodies against neurofilament and synaptophysin (in green). 